covered by water ice [Pilcher et al., 1972]. No evidence of gaseous absorption was detected [Fink et al., 1973], but the occultation experiment on Pioneer 10 indicated the presence of a very thin atmosphere on Io [Kliore et al., 1974a, b]. This satellite was also found to be the source of intense sodium emission [Brown, 1973] and seems to be surrounded by a cloud of sodium atoms whose distribution, origin, and mode of excitation are all current topics of active research [Trafton et al., 1974; McElroy et al., 1974; Fanale et al., 1974; Wamsteker et al., 1974].

A new satellite in the Jovian system was discovered by Kowal [1974]; it appears to be a member of the middle group with direct orbits. The Doppler tracking data from the Pioneer 10 spacecraft have been analyzed to derive masses for the Galilean satellites. The results indicate that two groups are present: Io and Europa with $\bar{\rho} \sim 3.5$ and Ganymede and Callisto with $\bar{\rho} \leqslant 2$ [Anderson el al. 1974].

The discovery by Kuiper [1952] that the particles in the rings of Saturn either are coated with water ice or actually consist in part of snowballs has been confirmed by recent work which has eliminated the possibility that detectable amounts of frozen ammonia might also be present [Pilcher et al., 1970; Kuiper et al., 1970]. Yet the ultraviolet reflectivity of the rings, which is remarkably similar to that of Titan [Barker and Trafton, 1973], requires that a nonicy component be present as well [Lebofsky et al., 1970]. A new inner ring was discovered by Guerin [1970].

The rings have been found to be unusually good reflectors of radar waves [Goldstein and Morris, 1973], a result that is still in the process of interpretation [Pollack et al., 1973; Pettengill and Hagfors, 1974; Briggs, 1974a].

\title{
A Review of Lunar Sample Studies and Their Application to Studies of the Terrestrial Planets
}

\author{
Arden L. Albee \\ Division of Geological and Planetary Sciences, California Institute of Technology, Pasadena, California 91125
}

\begin{abstract}
During the last half decade, hundreds of scientists from many countries have been studying the samples, photographs, and instrumental data returned from the moon by the Apollo and Luna programs. These studies have placed significant limits on chemical, petrologic, and physical parameters, on the time of many events, and on the rate of many processes and are giving greater insight into the natural processes that formed the moon and shaped its surface. Increasingly, it is being recognized that very similar processes governed the origin and evolution of planetary bodies throughout the solar system. Spacecraft have extended our sensors to all the terrestrial planets, and the insights gained from Apollo dominate our interpretation of the photographic and instrumental data returned from these bodies.
\end{abstract}

A major accomplishment of the Apollo and Luna programs is the transformation of planetary science from a discipline with important but untestable ideas to one in which the questions are both important and testable. Measurements on returned lunar samples, surface observations by astronauts, data from orbital and surface instruments, and orbital photographs have settled generations old controversies, which were stimulated by the extreme positions taken by men with equally great scientific intuition and eloquence but few facts.

Some earlier speculations have been contradicted and are now forgotten, while others have been made to look like axiomatic truths. Almost forgotten now is the intensity of the controversy on such subjects as the "cold old Copyright 1975 by the American Geophysical Union. moon' versus the 'hot young moon,' the depth to which a spacecraft might sink into uncompacted dust, the volcanic origin of craters and mare, the importance of water in shaping the mare and producing other topographic features, whether accumulated radiation would be released explosively as the astronaut touched the lunar surface, the presumption of a constant cratering rate throughout lunar history, the possibility of exotic lifeforms at depth in the drill cores even when none had been found in surface samples, the assertions that the dark color of certain lunar mountains is due to the abundance of carbon and that tectites come from the moon, etc. The answers may now seem obvious to the young scientists of today, but such controversies consumed countless manhours and thousands of printed pages during Apollo planning.

The flood of published lunar research has inundated even those scientists actively involved in lunar studies. In this review article, neither an attempt at a comprehensive listing of the papers nor a highly condensed survey of the research seems useful. A number of comprehensive papers have appeared recently which collectively provide both an excellent synthesis of lunar science and a good entrée into the important literature. Hence this article will review a selected group of these papers.

Four of the selected papers can be combined to provide a comprehensive and generally acceptable model for the formation and evolution of the moon. Although the four papers were authored by geophysicists, geochemists, and petrologists, they are basically compatible and interdepen- 
dent, each making effective use of the available chemical, isotopic, petrologic, geochronologic, geologic, and geophysical constraints. The selections include papers on the structure of the moon by Toksöz et al. [1974], on the thermal evolution of the moon and the terrestrial planets by Toksöz and Johnston [1975], on the geochemical evolution of the moon by Taylor and Jakes [1974], and on the origins of lunar rocks by Hays and Walker [1975].

The Toksöz et al. [1974] paper on the structure of the moon contains a comprehensive review of the seismic data. The velocity structure and attenuation properties of the lunar interior serve to characterize the moon as a differentiated body with a crust about $60 \mathrm{~km}$ thick, an elastic mantle about $1000 \mathrm{~km}$ thick, and an attenuating, perhaps partially molten, interior zone about $700 \mathrm{~km}$ thick. Density models are calculated by using the moment of inertia, mean density, and temperature and pressure dependency of the density for likely lunar materials. The models, assuming a chemically and mineralogically homogeneous mantle, are consistent with an olivinepyroxene mantle (but do not exclude other compositions) as well as with an $\mathrm{FeS}$ core with a maximum radius of 700 $\mathrm{km}$ or with an Fe core with a maximum radius of $450 \mathrm{~km}$. However, neither the presence nor the absence of a core can be demonstrated from the existing seismic and gravity data.

Many aspects of the evolution of a planetary bodydifferentiation, volcanism, tectonic activity, and magnetic fields-are controlled by its internal temperature and thermal history. Calculation of a realistic thermal evolution model requires very strong constraints from many kinds of data. Toksöz and Johnston [1975] have calculated the thermal evolution of Mars, Venus, Mercury, and the moon, starting from cosmochemical condensation models and using an assortment of geological, geochemical, and geophysical data to constrain both the present-day temperature profiles and the thermal histories of the planets interiors. Data and samples from the Apollo mission impose particularly strong constraints on the thermal evolution of the moon, and these are carefully discussed and documented in this paper. Lunar heat flow, electrical conductivity, seismic velocities and attenuations at various depths, lunar viscosity, seismicity, and tectonism provide evidence of the present-day temperatures and are manifestations of the internal structure. Magnetized lunar rocks, the differentiated lunar crust, the abundance of heat-producing elements, and the chronology of lunar magmatic activity indicate the thermal state earlier in lunar history. The calculated thermal models include sufficient melting to account for the extensive differentiation and upward concentration of radioactive heat sources early in lunar history. As the overlying lithosphere thickened, the top of the zone of extensive melting deepened with time, and the zone disappears a short time after the youngest-known mare filling. According to the model, the moon is cooling at present, but the deep interior may still be partially molten.

Such thermal models, constrained by the lunar data, can be useful tools for examining the effect of planetary size on the duration of high tectonic activity. This model predicts that the moon and Mercury are probably both inactive at the present time. Mars, intermediate in size, is probably still moderately active, whereas Venus may to- day have tectonic processes similar to those occurring on the earth.

Taylor and Jakês [1974] have combined data on the major, minor, and trace element chemistry with petrologic, geochronologic, and geophysical constraints into a comprehensive model for the geochemical evolution of the moon. A more extended consideration and compilation of supporting data will be in the forthcoming book Lunar Science: A Post-Apollo View [Taylor, 1975]. This model has received general acceptance by many lunar scientists, although probably none of them would agree with all details, and is providing a framework for the discussion and testing of new ideas.

The model assumes, and the paper argues, that the moon accreted from volatile-depleted refractory material, that the accretion was homogeneous, and that accretional melting involved most or all of the moon. Crystallization formed a chilled surface layer underlain by (1) an anorthite rich cumulate layer, (2) a layer containing residual liquid rich in $\mathrm{Ba}, \mathrm{U}, \mathrm{K}, \mathrm{REE}, \mathrm{P}, \mathrm{Th}$, etc., and (3) a pyroxene-olivine-spinel rich cumulate layer, resulting in a moon systematically zoned in both major and minor elements. During the first half-billion years of lunar history, intense meteorite bombardment pulverized and mixed the upper zones to great depths. The residual liquid-containing layer may be mixed upward by magmatic intrusion or mechanically by impact events. From 3.8 to 3.6 eons the Ti rich mare basalts were derived from a zone rich in $\mathrm{Fe}-\mathrm{Ti}$ oxides, and from 3.4 to 3.2 eons the quartz-or olivine- normative basalts were derived from a deeper layer. With time the depth to the zone of partial melting increased, and eventually, cessation of lunar volcanism resulted.

Hays and Walker [1975] use high-pressure phase equilibrium studies on lunar igneous rocks to provide information on mineral assemblages and mineral chemistry as functions of depth in the lunar interior. As in the previous models, they accept the evidence that the moon underwent accretional melting and that at least its outer portion consists of layers of cumulus crystals combined with crystallization products from trapped residual melts. They infer that the moon has a crust consisting of cumulus plagioclase with entrapped olivine, low-Ca pyroxene, and $\mathrm{Mg}-\mathrm{Al}$ rich spinel. The crust overlies a layered cumulate of olivine, low- and high-Ca pyroxene, and $\mathrm{Fe}$ and $\mathrm{Cr}$ rich spinel, separated by a zone rich in Fe-Ti oxide and crystallization products of residual melt. From 4.6 to 3.8 eons, lunar petrologic history was dominated by impact brecciation, metamorphism, and intracrustal partial melting at depths of less than $60 \mathrm{~km}$ of high-Al (plagioclase plus olivine plus low-Ca pyroxene or spinel) rocks. From 3.8 to 3.6 eons, Ti rich mare basalts were produced from partially melted $\mathrm{Ti}$ rich (olivine, low-Ca pyroxene, ilmenite, and spinel) rocks at depths of about $100 \mathrm{~km}$. From 3.4 to 3.2 eons, low-Ti mare basalts, produced from partially melted olivine pyroxenite at depths of $200-400 \mathrm{~km}$, reached the surface after varied degrees of crystal fractionation while moving upward. Present-day melting is limited to deeper depths, and lavas younger than 3.0 eons have not been identified. It is notable that this inverse correlation of experimentally inferred source depths for magma generation with the crystallization age of the resulting igneous rocks is compatible with the calculated thermal evaluation model of Toksöz and Johnston [1975]. 
$\mathrm{Rb}-\mathrm{Sr}$ mineral isochrons not only provide the current basic chronology of lunar evolution utilized in all the models discussed but impose rigorous constraints on lunar petrogenetic models. Albee and Gancarz [1975] summarize these data, discuss the isotopic evidence for large-scale differentiation at about 4.6 eons, and show that in most rocks, only very limited fractionation occurred during the subsequent evolution of most lunar rocks. These constraints are generally compatible with, although not explicitly included in, the preceding models.

An important lunar problem, one with implications for the study of other planets and of the sun's energetic particle history, involves the development of the lunar regolith. Models utilizing the measured micrometeorite flux have resulted in rates and mixing characteristics quite different than those for models utilizing measured abundance of neutron or cosmic ray produced isotopes. Resolution of these differences is necessary before the full potential of the core samples can be utilized to extend the record of the cosmic ray and high-energy particle flux back in time.

Walker [1975] summarized the major findings on the interaction of energetic particles with the lunar surface and on the problems of lunar surface dynamics. Hörz et al. [1975] discuss the observational evidence of lunar microcraters and utilize Monte Carlo and probabilistic approaches to gain insight into regolith development. They conclude that the regolith has to be envisioned as a sequence of discrete ejecta blankets in which localized mixing and homogenization by meteoritic flux occurred only in a surface layer about $1 \mathrm{~mm}$ thick prior to its being covered by a new ejecta layer. In general, the distribution of isotopes produced by energetic particles in individual regolith and core samples has been explained on an ad hoc basis rather than from a general statistical mixing model.

Remote sensing experiments have extended our knowledge of the moon's surface away from the ground truth afforded by the Apollo landing sites. Trombka et al. [1975] summarized the results from the orbital gamma ray and $X$ ray experiments. Elemental abundances for $\mathrm{Th}$, $\mathrm{U}, \mathrm{K}, \mathrm{Fe}, \mathrm{Ti}, \mathrm{Mg}, \mathrm{Al}, \mathrm{Si}$, and $\mathrm{O}$ have been determined over about $10 \%$ of the moon's surface. The highlands have a higher $\mathrm{Al} / \mathrm{Si}$ and a lower $\mathrm{Mg} / \mathrm{Si}$ than the mare, and the sharpness of compositional changes places strong constraints on the extent of horizonal transport processes. Localized areas of high radioactivity have been located.

McCord and Adams [1975] review studies relating the optical properties of returned lunar samples with those of 10 - to $20-\mathrm{km}$-diameter areas on the moon measured with earth-based telescopes. The wavelength dependence of reflected solar illumination provides quite specific information about the composition and mineralogy of surface materials such as are likely to compose the surfaces of many solar system objects. For example, Vidicon imaging devices have made it possible to map the distribution of the orange glass at the Apollo 17 site by means of its peculiar spectral properties. These results indicate that much of the moon's surface can be mapped from the earth and from orbit in terms of unique spectral properties of pyroxenes and glass.

It is clear that the surface composition of other planets can also be mapped by remote sensing experiments and that the moon, with the ground truth provided by Apollo, will provide useful tests for these instruments.
Much as Toksöz and Johnston [1975] extended their thermal evolution model from the moon to the terrestrial planets, Ganapathy and Anders [1974] have proposed a generalized accretion-condensation model. If this model is correct, it permits the determination of the bulk composition of a planet from a limited number of observations. The bulk compositions of the earth and moon were calculated in terms of seven components: early refractory condensate, FeS, remelted and unremelted Fe-Ni-Co and (Mg, Fe) silicates, and carbonaceous volatile-rich silicates. The proportions of these components in a differentiated body may be estimated from geochemical constraints, e.g., bulk $\mathrm{U}$ content of the planet based on the heat flow, bulk Fe of the planet based on the density, and $\mathrm{K} / \mathrm{U}, \mathrm{FeO} / \mathrm{MnO}$, and $\mathrm{Tl} / \mathrm{U}$ values for surface rocks. The model permits calculation of the abundance of 82 elements from six measurements, which could be accomplished by unmanned instruments.

Prinz et al. [1975] have drawn attention to marked petrographic similarities between lunar and meteoritic rocks. Increased understanding of such features in both lunar and meteoritic samples is increasing the usefulness of meteorites as probes for the early history of the solar system. For example, the nature of rock fragments in the lunar regolith has proved useful in providing petrologic and chemical characterization of the rock units in the vicinity of each landing site. Recognition that certain meteorites evolved in an impact-derived regolith on their parent body makes it logical to use the same approach in order to increase our understanding of the planetesimals in the solar system.

To understand the evolution of planetary interiors and surfaces, it is necessary to establish a time scale to which major events in a planet's history can be referred. Wetherill [1975] has approached this problem by combining lunar chronologic data with orbital dynamic studies. Possible sources were evaluated for both the 'normal,' presumably rapidly declining, impact flux during the first 600 m.y. of lunar history and for a possible 'catastrophic' flux about 4.0 eons ago. His studies indicate that the most likely sources would probably produce a history of largescale impacts on the earth, Mercury, and Venus similar to that observed and dated on the moon. Thus establishment of history of the impact flux for the moon represents a major step in establishing a cratering time scale for all the inner planets and in understanding the early history of the earth.

Prior to Apollo it seemed unlikely that lunar magnetism would be of much interest, since Explorer 35 detected no planetary-wide magnetic field at all comparable to that of earth. However, Apollo studies established the presence of natural remanent magnetism in returned samples, and the presence of anomalies ranging from 2 to $200 \mathrm{~km}$ in size. Fields 20-200 times greater than the field in the solar wind are required to generate the observed remanent magnetism, but as yet, the origin of the lunar remanent fields remains an enigma. Dyal et al. [1974] have summarized these data and have calculated lunar magnetic permeability, bulk lunar iron abundance, lunar electrical conductivity, and a lunar interior temperature profile.

The interpretations by the team studying the Mercury photographs are greatly enhanced by the now highly evolved understanding of lunar evolution. A photogeologic sequence, similar to the sequence for the moon as known 
before Apollo, has been determined from the Mariner 10 television pictures. This sequence has been interpreted in a series of episodes like those in the evolution of the moon [Murray et al., 1974]. These episodes are (1) accretion, extensive differentiation, and development and loss of any atmosphere, (2) a period of intense bombardment, including formation of basins as large as $1300 \mathrm{~km}$ in diameter, (3) filling of the basins and extensive flooding, at least in part, by volcanic rocks, and (4) light cratering and development of a lunarlike regolith. Considerations similar to those of Wetherill [1975] further suggest that the events recorded on Mercury's surface have ages similar to those already dated geochronologically on the lunar surface.

The details of this evolutionary sequence are clearly dependent on Apollo results and stand in sharp contrast to the pre-Apollo interpretations of the similar photogeologic sequence on the moon. That sequence was interpreted by two common, nearly opposing pre-Apollo schools of thought: (1) the moon was geologically active throughout most of its history, or (2) the moon was essentially dead shortly after its formation.
Many of the papers selected for review here have a common characteristic-they are written by scientists who, during Apollo, were almost totally involved in making measurements and obtaining data and who, post-Apollo, are themselves synthesizing the results into general models applicable to other planets as well as the moon. Four years ago in a similar review article [Albee, 1971] I concluded '. . . the Apollo samples have given a vast new insight into the processes that formed the moon and shaped its surface. The results do not resolve the problem of the origin of the moon. However, significant limits have been established for the chemical parameters, on the time, and on the rate of many processes.'

Where the moon originated is still not resolved, but it is certain that lunar science has 'given a vast new insight into the processes that formed' all the terrestrial planets and shaped their surfaces.

Acknowledgment. Contribution 2567 of the Division of Geological and Planetary Sciences, California Institute of Technology, Pasadena, California.

\title{
Cosmogony of the Solar System
}

\author{
William R. Ward \\ Center for Astrophysics, Harvard College Observatory and Smithsonian Astrophysical Observatory \\ Cambridge, Massachusetts 02138
}

This report is intended to cover research on solar system origin over the last 4 years. Emphasis has been placed on the physics of planetary accumulation mechanisms, although pertinent references on the chemistry of mineral condensation and cometary research are included.

\section{Solar Nebula}

The most ambitious attempt to model the solar nebula to date is the 1973 paper of Cameron and Pine [1973]. This model is characterized by a large mass $\left(-2 M_{S}\right)$ and is modeled as a collapse of spherical distribution of gas with an angular momentum per unit mass estimated from the assumption of fully developed turbulent motion. A disk structure is determined by requiring a radial and vertical balance of forces, i.e., gravitational, centrifugal, and pressure gradient forces. The most striking and controversial feature of the model is the absence of a central concentration of material representing the protosun. Rather, the sun forms as a secondary process of angular momentum redistribution via Eddington-Sweet type circulation currents during which the inner portion of the nebula contracts and the outer portion expands radially. The large Copyright ${ }^{\oplus} 1975$ by the American Geophysical Union. mass of the system is justified on two counts: there must be sufficient material to allow angular momentum redistribution, and a $T$-tauri phase has presumably ejected a few tenths of a solar mass from the system. Another noteworthy attribute of the model is the presence of a vertical convection zone between $\sim 1$ and $\sim 10$ AU due to the opacity of condensed iron grains.

A serious obstacle faced by the Cameron-Pine model is the question of its overall stability. The self-gravity of a nebula this large is by no means negligible, and there is a tendency for this disk to fragment, either by secular instability with a barlike mode or by dynamic instability [Ostriker, 1973]. A large central condensation, such as the protosun, would lend stability to the structure. However, prior to the contraction of the inner nebula to form the sun, stability is marginal.

\section{Chemical Condensation}

Work has continued on the condensation sequence for a cooling gas of solar composition. Lewis [1972a] has investigated low-temperature condensates under the alternative assumptions that the accretion time is much shorter or much longer than the nebula cooling time. Grossman 